\title{
Nontraumatic Fat Embolism Found Following Maternal Death after Cesarean Delivery
}

\author{
Tabitha Schrufer-Poland, MD, $\mathrm{PhD}^{1}$ \\ Dev Maulik, MD, PhD 13,3 \\ Paul Singh, $M D^{2}$ \\ ${ }^{1}$ Department of Obstetrics and Gynecology, Kansas City School of \\ Medicine, University of Missouri, Kansas City, Missouri \\ 2 Division of Maternal-Fetal Medicine, Department of Obstetrics and \\ Gynecology, Kansas City School of Medicine, University of Missouri, \\ Kansas City, Missouri \\ ${ }^{3}$ Department of Obstetrics and Gynecology, Truman Medical Center, \\ Kansas City, Missouri \\ ${ }^{4}$ Department of Maternal Fetal Medicine, Children's Mercy Hospital, \\ Kansas City, Missouri
}

Am J Perinatol Rep 2015;5:e1-e5.
Cristiano Jodicke, $\mathrm{MD}^{2}$
Sara Reynolds, MD ${ }^{1}$

\author{
Address for correspondence Tabitha Schufer-Poland, MD, PhD, \\ Department of Obstetrics and Gynecology, Kansas City School of \\ Medicine, University of Missouri, 2301 Holmes Street, \\ Kansas City, MO 64108 (e-mail: schruferpolandt@umkc.edu).
}

\begin{abstract}
Keywords

- pregnancy

- fat embolism

- maternal death

- cesarean section

Introduction Fat embolism is a rare form of nonthrombotic embolization. Limited literature exists regarding the diagnosis of fat embolism during the perinatal period. We present the first case of maternal death that resulted from nontraumatic fat embolization following Cesarean delivery.

Case Description A 29-year-old gravida 1 with a complex medical and surgical history underwent a primary Cesarean delivery at term. On postoperative day 2 the patient was found to be unresponsive. Despite resuscitative efforts, the patient succumbed. Autopsy findings were remarkable for diffuse pulmonary fat emboli. Furthermore, there was no histological evidence of either amniotic fluid embolism or thromboembolism. The primary cause of death was attributed to nontraumatic fat embolization.

Discussion Multiple risk factors may have contributed to the development of nontraumatic fat embolization in our patient. Obstetricians should maintain a high level of suspicion for nontraumatic fat embolization in cases of maternal respiratory decompression and sudden maternal mortality.
\end{abstract}

Fat embolism is a rare form of nonthrombotic embolization that is most often associated with blunt force trauma of the pelvis or lower extremity long bones. ${ }^{1}$ However, cases of nontraumatic fat embolism (NTFE) have also been described in association with joint arthroplasty, ${ }^{2}$ lipid emulsion infusion in total parenteral nutrition, ${ }^{3-5}$ sickle cell crisis, ${ }^{6-9}$ prolonged corticosteroid administration, ${ }^{10,11}$ and fatty liver disease. ${ }^{12-14}$ Apart from pulmonary complications that are often seen in cases of embolic disorders, both traumatic and nontraumatic fat emboli may be associated with the development of respiratory, cutaneous, and neurologic symptoms. Although, extremely rare in pregnancy, isolated cases of perinatal fat embolism have been reported to occur. ${ }^{15-18}$ Herein, we report the first case of a maternal death that occurred as a result of NTFE following a Cesarean delivery.

\section{Case Description}

A 29-year-old primigravida presented to our high-risk obstetrical clinic at 18 gestational weeks with a complex medical history significant for Arnold-Chiari malformation, hydrocephalus, spina bifida with tethered cord syndrome, neurogenic bladder, and chronic decubitus ulcerations as a result of prolonged immobility. Her surgical history consisted of received

June 25, 2014 accepted after revision

August 28, 2014

published online

December 17, 2014
DOI http://dx.doi.org/

10.1055/s-0034-1394153. ISSN 2157-6998.
Copyright $\odot 2015$ by Thieme Medical Publishers, Inc., 333 Seventh Avenue, New York, NY 10001, USA. Tel: +1(212) 584-4662.
License terms

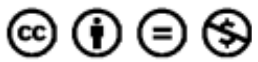


ventriculoperitoneal shunting, multiple lumbosacral laminectomies, numerous irrigation, and debridement for perineal ulcerations eventually necessitating a diverting colostomy and an ileovesicostomy. As the patient had minimal support infrastructure, a social service consultation was made to assist with nursing home placement and transportation to her outpatient appointments. The patient declined genetic screening and her initial prenatal laboratory values, fetal anatomy survey, and serial fetal growth evaluations were normal. At 28 gestational weeks, she developed gestational diabetes and was successfully treated with oral hypoglycemic agents. Her antepartum surveillance consisted of weekly biophysical profiles beginning at 28 gestational weeks which were reassuring. At 38 weeks, the patient developed mild preeclampsia that necessitated delivery. Due to the inability to provide the patient with regional anesthesia due to her history of complex spinal surgery coupled with the difficulty in maintaining dorsal lithotomy position through the second stage of labor as a result of her lower extremity contractures and the patient's desire to avoid a vaginal delivery in the absence of reliable pain control, a Cesarean delivery under general anesthesia was undertaken. The neonate was delivered without complications and the remainder of the Cesarean section was uneventful. On postoperative day 1 the patient was alert and responsive as well as tolerating medications and fluids by mouth. Although the patient's blood pressure remained mildly elevated, she denied any other symptoms of preeclampsia and had normal laboratory values.
On the morning of postoperative day 2 , however, the patient was found to be unresponsive, without an obtainable pulse or blood pressure. Bilateral intraosseous lines were placed and cardiopulmonary resuscitation (CPR) was initiated. Despite several cycles of CPR, no response was obtained and the patient was pronounced dead. Autopsy examination revealed pulmonary vasculature with optical clearing of the lumens suggestive of fat emboli (-Fig. 1A). The Oil Red $O$ stain showed scattered fat droplets in alveolar spaces and distal, smaller blood vessels in different areas throughout the lungs (-Fig. 1B). Immunohistochemical stain for pan-cytokeratin and special staining for $\mathrm{c}$ were performed on lung sections and were negative for fetal squamous cells or mucinous debris which are often associated with amniotic fluid embolism (-Figs. 2 and $\mathbf{3}$ ). The remainder of the autopsy findings were unremarkable, including no evidence of rib or long bone fractures, amniotic fluid embolism, or thromboembolism. The cause of death was attributed to multiple nonthrombotic pulmonary fat emboli.

\section{Discussion}

First described by Zenker in 1862, fat embolism occurs with a frequency of 3 to $4 \%$ in the United States. Following the onset of respiratory distress, pulmonary fat emboli often present with hypoxia, tachypnea, and tachycardia. Increased pulmonary vasoconstriction, vascular resistance, and hypertension, then occur, followed by right-sided heart failure, pulmonary
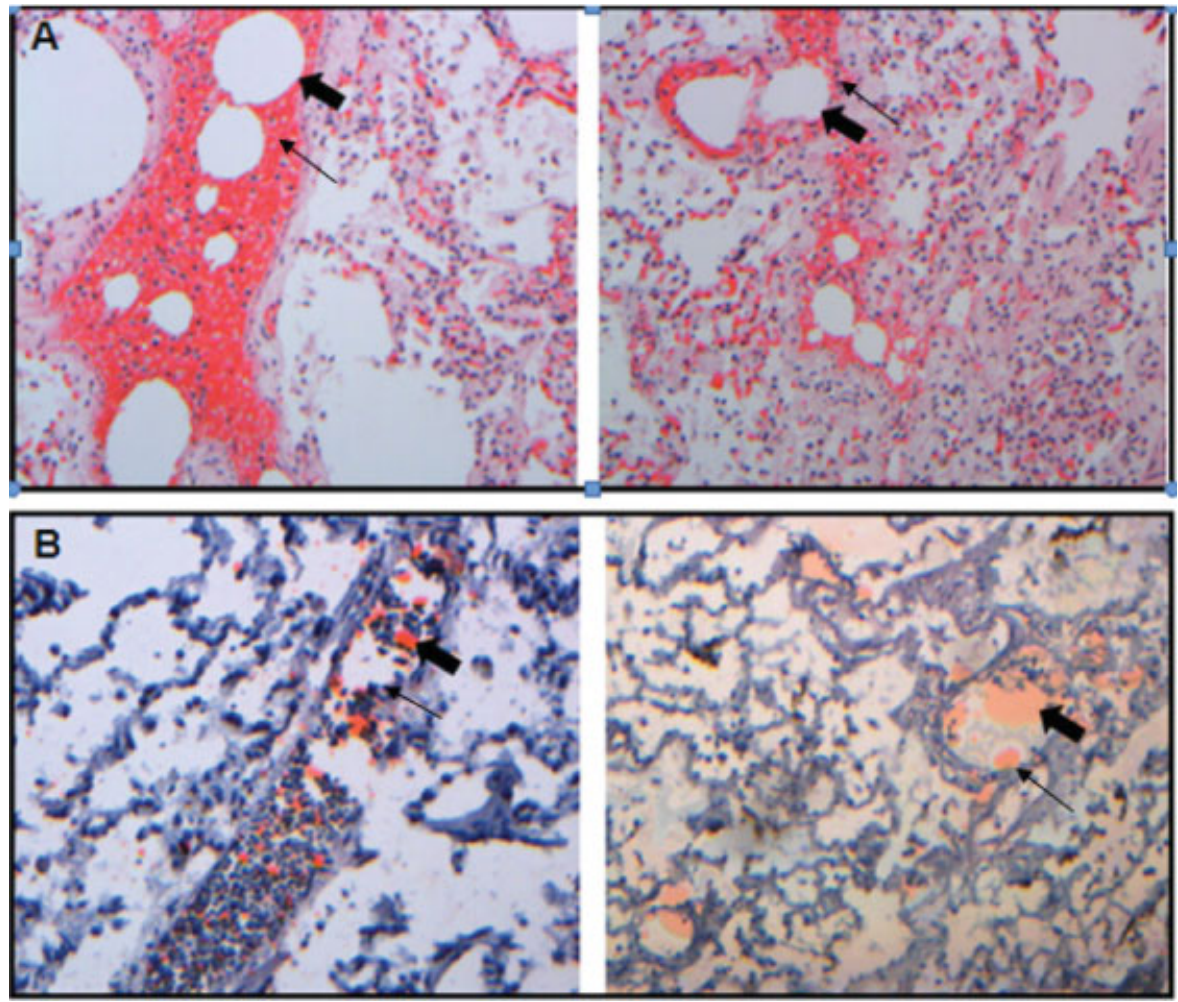

Fig. 1 (A) Hematoxylin and eosin stain demonstrating absence of staining of intravascular fat (block arrows) in sagittal (left) and transverse (right) sections of the pulmonary parenchyma and microvasculature, magnified to $\times 100$. (B) Oil Red $O$ stain highlighting intravascular fat that appear orange (block arrows) in sagittal (left) and transverse (right) sections of the pulmonary parenchyma and microvasculature, magnified to $\times 100$. Note that in all sections the lined arrows represent the vascular wall. 


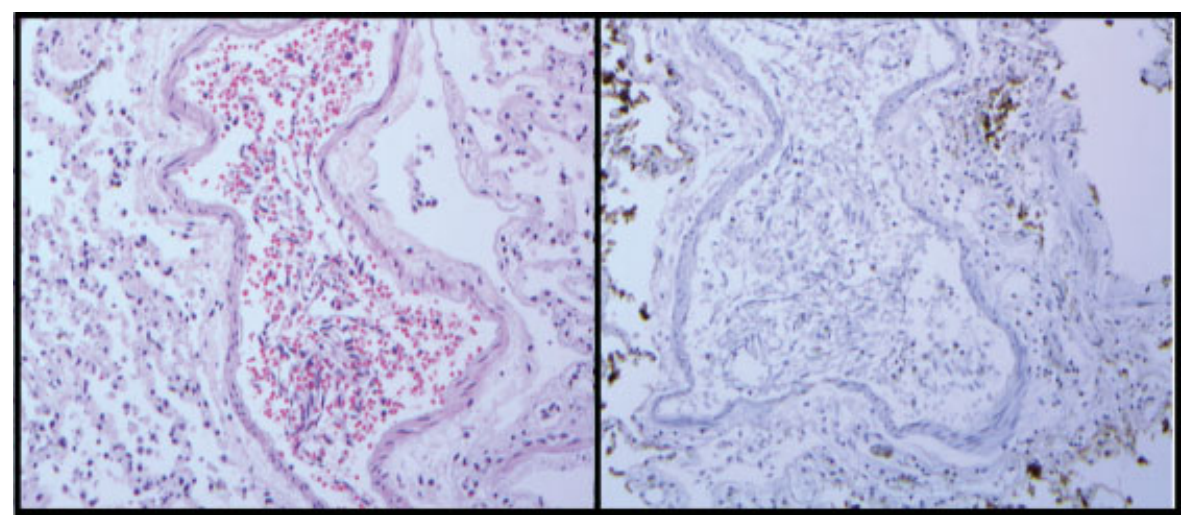

Fig. 2 Hematoxylin and eosin stain of transverse sections of the pulmonary parenchyma with arterioles containing intravascular blood elements and intracellular debris, magnified to $\times 20$ (left), special stain with pan-cytokeratin demonstrating absence of squamous cells within the pulmonary vasculature, magnified to $\times 20$ (right).

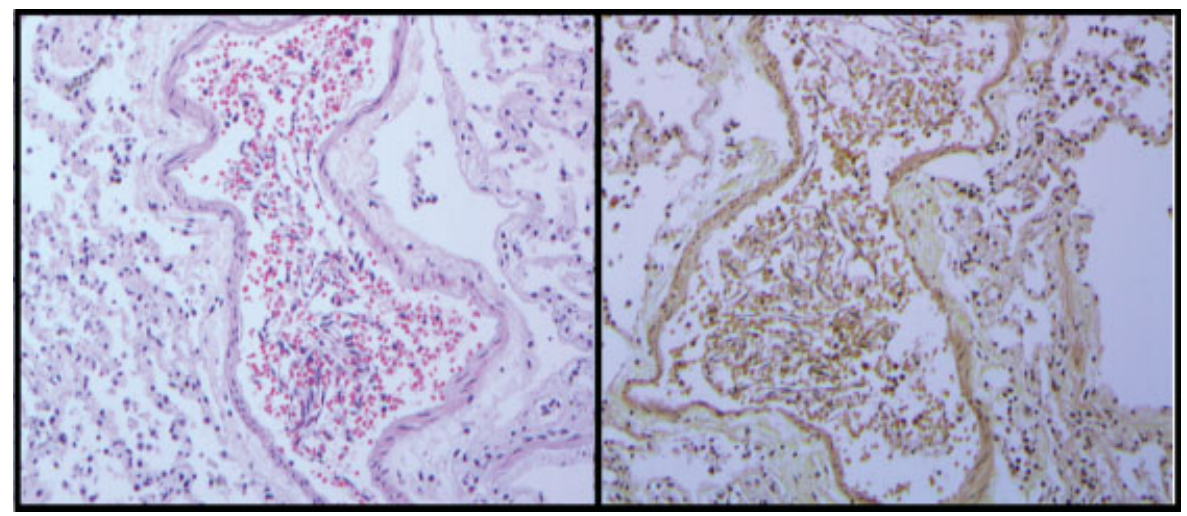

Fig. 3 Hematoxylin and eosin stain of transverse sections of the pulmonary parenchyma with arterioles containing intravascular blood elements and intracellular debris, magnified to $\times 20$ (left), special stain with mucicarmine demonstrating absence of mucous within the pulmonary vasculature, magnified to $\times 20$ (right).

edema, left ventricular dysfunction, and finally death. Apart from the cardiac and respiratory consequences, patients with fat emboli can have dermatologic sequelae, including reddish-brown nonpalpable petechiae over the upper body as well as subconjunctival, oral, and retinal petechiae. In addition, fat emboli may cause patients to develop altered mental status or seizure activity.

Risk factors for the development of pulmonary fat embolism include traumatic etiologies such as fractures to the ribs or long bones, recent orthopedic surgery as well as nontraumatic etiologies such as parenteral lipid infusion, immunosuppression from recent corticosteroid exposure or diabetes mellitus, burns, liposuction, cardiopulmonary bypass, and sickle cell anemia with acute chest syndrome. Unfortunately, the etiology of fat embolism is frequently unclear, leading to difficulty in ascertainment of primary cause of death, especially in the case of trauma sustained during resuscitative efforts. In one case series, review of, 50 autopsies over the course of a year in both trauma and nontrauma patients revealed evidence of fat embolism in $88 \%$ of nontrauma, and $86 \%$ of trauma patients who received cardiopulmonary resuscitation. ${ }^{19}$ Fat embolism, therefore, may be an incidental finding in patients receiving chest compressions. Further, it has been well documented that intraosseous access is associated with the development of fat embolism and has been reported in conjunction with patient mortality. ${ }^{20}$

Unfortunately, fat emboli are frequently underdiagnosed mainly as a result of the similarity of presenting symptoms associated with other etiologies of pulmonary emboli, an initial asymptomatic period of up to 72 hours, and the lack of sensitivity and specificity of laboratory and imaging techniques. Patients with fat emboli may develop thrombocytopenia, anemia, hypofibrinogenemia, or urinary fat globules. However, these findings are considered nonspecific and cannot be relied upon as confirmatory for the diagnosis of fat embolism. Pulmonary capillary cytology obtained from pulmonary artery catheterization may identify patients at risk for the development of pulmonary fat emboli. Unfortunately, the risks associated with pulmonary catheter placement often preclude the clinical utility of pulmonary capillary cytology for the diagnosis of fat emboli. Serial chest radiographs may reveal increasing bilateral pulmonary infiltrates within 2 days of the onset of clinical findings. While nuclear medicine ventilation-perfusion imaging of the lungs and helical chest computed tomography scans are frequently 
normal in patients with fat emboli, subsegmental perfusion defects, and ground glass opacities may be seen respectively. Bronchoalveolar lavage for the detection of fatty alveolar macrophages and lipid inclusion bodies has been utilized in trauma patients with mixed results. Histologically, fat embolism can be confirmed by optical clearing seen within the pulmonary vasculature after staining with Oil Red O. Differentiation from an amniotic fluid embolism may be made by the absence of fetal epithelial cells or mucinous debris within the pulmonary circulation. ${ }^{21}$

Medical care for fat embolism is mainly supportive and includes the maintenance of adequate oxygenation and ventilation as well as hemodynamic stability. Several studies have shown that early mobilization and prophylactic corticosteroid therapy in trauma patients to be at least moderately effective in reducing the incidence of pulmonary fat embolism. ${ }^{22-27}$ Schonfeld et al performed a randomized controlled trial in 64 patients with lower-extremity long-bone fractures. $^{11}$ Fat embolism was diagnosed in $22 \%$ of patients exposed to placebo versus none in the steroid-treated arm. The frequency of pulmonary embolization as a primary cause of death in nontrauma patients, and the inability to distinguish fat embolization from other causes of sudden cardiac and respiratory death has precluded the further investigation of preventive measures or treatment.

Although the exact pathophysiology of fat embolization has not been clearly delineated, several theories exist. The mechanical theory posits that disrupted fat cells from bone marrow or adipose tissue may enter damaged venules, gaining access to the systemic circulation. ${ }^{28}$ These lipids may then enter the arterial system via passage through a patent foramen ovale or via microembolism through the capillary beds in the lungs. The biochemical theory suggests that toxic metabolites of endogenous lipids in the plasma, such as free fatty acids and C-reactive protein accumulate under periods of stress and catecholamine release. ${ }^{29,30}$ These acute phase reactants can then result in either direct tissue damage, resulting in acute lung injury, acute respiratory distress syndrome, and cardiac dysfunction or indirectly cause the agglutination of chylomicrons, low-density lipoproteins, and liposomes that can ultimately systemically embolize as described according to the mechanical theory.

There has been a paucity of literature regarding fat emboli diagnosed during the perinatal period. Karayel et al, in 2005, was the first to associate the pathologic findings of a fat embolism with maternal death. ${ }^{31}$ A previously healthy patient became unresponsive and developed respiratory distress followed by respiratory arrest 4 hours after an uneventful spontaneous vaginal delivery. Despite initial successful resuscitation, she underwent a second episode of respiratory arrest 2 hours later and succumbed. Autopsy findings were consistent with severe diffuse pulmonary fat emboli and the cause of death was attributed to intractable respiratory failure. Prenatal fat embolism has been reported to occur in patients with hemoglobinopathies ${ }^{15,16}$ and acute fatty liver of pregnancy. ${ }^{17,18}$

We propose that in our case the combination of pregnancy, gestational diabetes, and prolonged immobilization contrib- uted to an increased risk of NTFE. In addition, the manipulation of subcutaneous and intraperitoneal adipose tissue as well as the stress of surgery incurred during Cesarean delivery provided additional risk factors for NTFE. Caution, however, must be applied with regards to the ascertainment of the final cause of death since the diagnosis of fat embolism may have been influenced by the performance of CPR and the placement of intraosseous access. Nonetheless, findings of sudden maternal respiratory decompensation should prompt obstetricians to maintain a high level of suspicion for fat embolization. Furthermore, unexplained maternal mortality should always warrant further pathologic diagnosis of fat embolization via autopsy.

\section{Disclosure}

No competing financial conflicts exist for any author/ investigator.

\section{References}

1 Mudd KL, Hunt A, Matherly RC, et al. Analysis of pulmonary fat embolism in blunt force fatalities. J Trauma 2000;48(4):711-715

2 Ahmadzai H, Campbell S, Archis C, Clark WA. Fat embolism syndrome following percutaneous vertebroplasty: a case report. Spine J 2014;14(4):e1-e5

3 Barson AJ, Chistwick ML, Doig CM. Fat embolism in infancy after intravenous fat infusions. Arch Dis Child 1978;53(3):218-223

4 Levene MI, Wigglesworth JS, Desai R. Pulmonary fat accumulation after intralipid infusion in the preterm infant. Lancet 1980; 2(8199):815-818

5 Levine M, Skolnik AB, Ruha AM, Bosak A, Menke N, Pizon AF. Complications following antidotal use of intravenous lipid emulsion therapy. J Med Toxicol 2014;10(1):10-14

6 Horton DP, Ferriero DM, Mentzer WC. Nontraumatic fat embolism syndrome in sickle cell anemia. Pediatr Neurol 1995;12(1):77-80

7 Garza JA. Massive fat and necrotic bone marrow embolization in a previously undiagnosed patient with sickle cell disease. Am J Forensic Med Pathol 1990;11(1):83-88

8 Vichinsky E, Williams R, Das M, et al. Pulmonary fat embolism: a distinct cause of severe acute chest syndrome in sickle cell anemia. Blood 1994;83(11):3107-3112

9 Eckardt P, Raez LE, Restrepo A, Temple JD. Pulmonary bone marrow embolism in sickle cell disease. South Med J 1999; 92(2):245-247

10 Rosen JM, Braman SS, Hasan FM, Teplitz C. Nontraumatic fat embolization. A rare cause of new pulmonary infiltrates in an immunocompromised patient. Am Rev Respir Dis 1986;134(4): 805-808

11 Schonfeld SA, Ploysongsang Y, DiLisio R, et al. Fat embolism prophylaxis with corticosteroids. A prospective study in highrisk patients. Ann Intern Med 1983;99(4):438-443

12 Walsh K, Alexander G. Alcoholic liver disease. Postgrad Med J 2000;76(895):280-286

13 Schulz F, et al. Fatal fat embolism in acute hepatic necrosis with associated fatty liver. Am J Forensic Med Pathol 1996;17(3):264-268

14 Randall B. Fatty liver and sudden death. A review. Hum Pathol 1980;11(2):147-153

15 Faron G, Corbisier C, Tecco L, Vokaer A. First sickle cell crisis triggered by induction of labor in a primigravida. Eur J Obstet Gynecol Reprod Biol 2001;94(2):304-306

16 Bódis L, Szupera Z, Pierantozzi M, et al. Neurological complications of pregnancy. J Neurol Sci 1998;153(2):279-293 
17 Jones JP Jr. Fat embolism, intravascular coagulation, and osteonecrosis. Clin Orthop Relat Res 1993;(292):294-308

18 Sinha A, Olah KS. Acute fatty liver of pregnancy: an unusual presentation. J Obstet Gynaecol 2005;25(1):60-61

19 Eriksson EA, Pellegrini DC, Vanderkolk WE, Minshall CT, Fakhry SM, Cohle SD. Incidence of pulmonary fat embolism at autopsy: an undiagnosed epidemic. J Trauma 2011;71(2):312-315

20 Hasan MY, Kissoon N, Khan TM, Saldajeno V, Goldstein J, Murphy SP. Intraosseous infusion and pulmonary fat embolism. Pediatr Crit Care Med 2001;2(2):133-138

21 Rath WH, Hoferr S, Sinicina I. Amniotic fluid embolism: an interdisciplinary challenge: epidemiology, diagnosis and treatment. Dtsch Arztebl Int 2014;111(8):126-132

22 Riska EB, Myllynen P. Fat embolism in patients with multiple injuries. J Trauma 1982;22(11):891-894

23 Alho A, Saikku K, Eerola P, Koskinen M, Hämäläinen M. Corticosteroids in patients with a high risk of fat embolism syndrome. Surg Gynecol Obstet 1978;147(3):358-362

24 Lindeque BG, Schoeman HS, Dommisse GF, Boeyens MC, Vlok AL. Fat embolism and the fat embolism syndrome. A double-blind therapeutic study. J Bone Joint Surg Br 1987;69(1):128-131
25 Kallenbach J, Lewis M, Zaltzman M, Feldman C, Orford A, Zwi S. 'Low-dose' corticosteroid prophylaxis against fat embolism. J Trauma 1987;27(10):1173-1176

26 Bederman SS, Bhandari M, McKee MD, Schemitsch EH. Do corticosteroids reduce the risk of fat embolism syndrome in patients with long-bone fractures? A meta-analysis. Can J Surg 2009;52(5):386-393

27 Kubota T, Ebina T, Tonosaki M, Ishihara H, Matsuki A. Rapid improvement of respiratory symptoms associated with fat embolism by high-dose methylpredonisolone: a case report. J Anesth 2003;17(3):186-189

28 Pell AC, Hughes D, Keating J, Christie J, Busuttil A, Sutherland GR. Brief report: fulminating fat embolism syndrome caused by paradoxical embolism through a patent foramen ovale. N Engl J Med 1993;329(13):926-929

29 Hulman G. Pathogenesis of non-traumatic fat embolism. Lancet 1988;1(8599):1366-1367

30 Nixon JR, Brock-Utne JG. Free fatty acid and arterial oxygen changes following major injury: a correlation between hypoxemia and increased free fatty acid levels. J Trauma 1978;18(1):23-26

31 Karayel F, Arican N, Kavas G, Turan AA, Pakis I. Maternal death due to non-traumatic fat embolism. J Forensic Sci 2005;50(5):1201-1203 